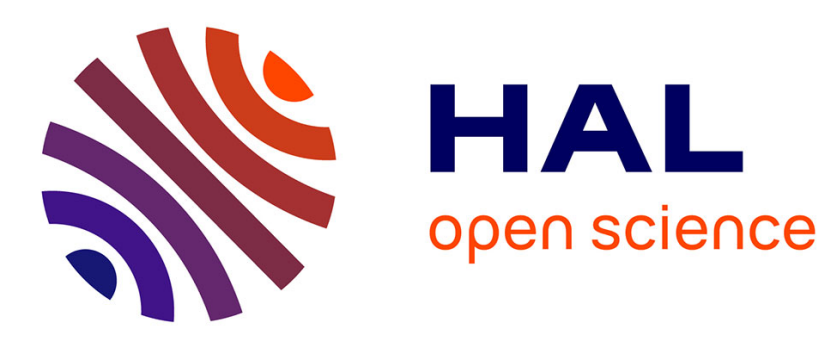

\title{
Identification of Discrete Event Systems: Implementation Issues and Model Completeness
}

\author{
Matthias Roth, Lothar Litz, Jean-Jacques Lesage
}

\section{To cite this version:}

Matthias Roth, Lothar Litz, Jean-Jacques Lesage. Identification of Discrete Event Systems: Implementation Issues and Model Completeness. 7th International Conference on Informatics in Control, Automation and Robotics (ICINCO), Jun 2010, Funchal, Portugal. pp.73-80. hal-00525602

\section{HAL Id: hal-00525602 \\ https://hal.science/hal-00525602}

Submitted on 12 Oct 2010

HAL is a multi-disciplinary open access archive for the deposit and dissemination of scientific research documents, whether they are published or not. The documents may come from teaching and research institutions in France or abroad, or from public or private research centers.
L'archive ouverte pluridisciplinaire HAL, est destinée au dépôt et à la diffusion de documents scientifiques de niveau recherche, publiés ou non, émanant des établissements d'enseignement et de recherche français ou étrangers, des laboratoires publics ou privés. 


\title{
IDENTIFICATION OF DISCRETE EVENT SYSTEMS: IMPLEMENTATION ISSUES AND MODEL COMPLETENESS*
}

\author{
Matthias Roth, Lothar Litz \\ Institute of Automatic Control, University of Kaiserslautern, Germany \\ mroth@eit.uni-kl.de,litz@eit.uni-kl.de \\ Matthias Roth, Jean-Jacques Lesage \\ LURPA, Ecole Normal Supérieur de Cachan, France \\ jean-jacques.lesage@lurpa.ens-cachan.fr
}

Keywords: Discrete Event Systems, Identification, Implementation

\begin{abstract}
This paper presents some practical issues for the identification of discrete event systems (DES). The considered class of systems consists of a plant and a controller running is a closed-loop. Special emphasis is given to a data collection procedure using industrial controllers and its impact on the external DES-behavior of the considered systems. For models identified on the basis of observed external DES behavior using the algorithm from (Klein, 2005) it is shown that under some conditions, the identified model language simulates the complete original system language even if only a subset of this language is available for identification. This model characteristic is crucial for many model-based techniques like diagnosis or verification. Analyzing the observed data of a laboratory facility it is shown how it can be decided if the conditions for a complete model hold for an existing application.
\end{abstract}

\section{INTRODUCTION}

Model-based techniques play a key role in many modern control applications. For systems that can be modeled as Discrete Event Systems (DES) various approaches to improve system dependability using model-based methods have been proposed in the last two decades. Examples for these methods are diagnosis (Sampath et al., 1996) and formal verification with model checking (Machado et al., 2006). A bottleneck for the application of model-based techniques is the process of model-building which is usually expensive due to high costs for the necessary specialists. A promising way to facilitate the use of model-based methods is to offer efficient identification methods in order to decrease the cost of model-building.

First approaches for the identification of DES have been proposed in the sixties and seventies of the last century in the field of computer science (Biermann and Feldman, 1972). The identification of physical systems which is a typical interest in many engineering domains is not the aim of these works. More recent works especially on identification of Petri nets

*This works was partially supported by a grant from Région Île-de-France are summarized in (Fanti and Seatzu, 2008). In the last years two main directions of constructing a Petri net from samples of its language have been followed: In the first class of approaches specific rules about interdependencies of observed events are used to identify a Petri net on the basis of observed firing and marking sequences (Meda-Campana and LopezMellado, 2005). The second class of approaches uses optimization techniques like integer programming to derive a Petri net structure according to given constraints (Giua and Seatzu, 2005), (Dotoli et al., 2006).

The main obstacle for the application of these methods to real world systems is their relatively high degree of abstraction. The work is usually not focused on questions like how to represent data that can be captured from a real system and how to cope with inadequacies inherent to the data collection process. In (Dotoli et al., 2006) a case study shows that there is a considerable potential of identification methods to obtain meaningful DES models of physical systems. Since the identification data base in this work has been obtained by simulating a three tank system, important issues of working with data captured from a real system have not been addressed.

In (Klein et al., 2005) an algorithm for the iden- 
tification of closed-loop DES is presented. The algorithm has been designed to work with data obtained from real systems and yields a monolithic automaton. In this paper some implementation issues concerning the application of this algorithm to a real system are presented. In section 2 the class of closed-loop DES is presented and it is outlined that this system class is an appropriate modeling formalism for many industrial systems. Section 3 summarizes some practical implications of the data collection procedure and defines an appropriate data format for the identification algorithm. The identification algorithm is compactly presented in section 4 . An important property of models identified with this algorithm is proofed in this section: Under some well-defined conditions concerning the observed system language, the identified model is able to simulate the complete original system language of arbitrary length ${ }^{1}$. In section 5 the data collection procedure and the identification algorithm are applied to a laboratory facility in order to show the relevance of the approach for real systems. It is shown how it can be decided of if the preconditions presented in the former section hold only using measured system data.

\section{CLOSED-LOOP DES}

A typical configuration of industrial systems is a closed-loop of controller and plant. In the plant, a set of sensors measures certain process values and delivers them to the controller using the controller inputs. The controller executes a control algorithm and determines appropriate actuator settings for the plant. Commands to actuators in the plant are transfered via controller output signals. Figure 1 shows this principle.

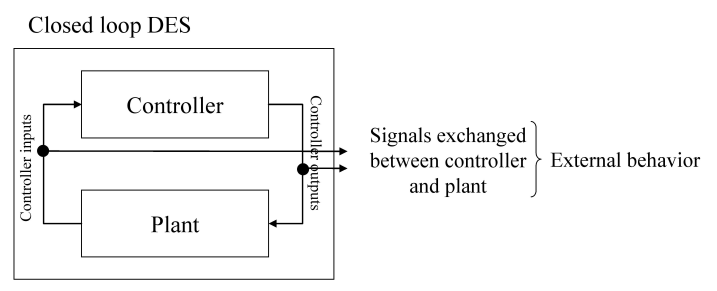

Figure 1: Closed-loop Discrete Event system

The external behavior of such systems can be obtained by an analysis of the signals exchanged between controller and plant. In the considered class of systems these signals are binary. From an external point of view, a signal changes its value asyn-

\footnotetext{
${ }^{1}$ Language $L_{A}$ simulates language $L_{B}$ if $L_{A} \supseteq L_{B}$ holds
}

chronously which can be considered as the occurrence of an event. The closed-loop system can be characterized as non-deterministic since it consists of the combination of a deterministic subsystem (controller) and a non-deterministic subsystem (plant). Since the closed-loop system does not have any inputs, it must be considered as an event generator (note that the controller inputs also belong to the external behavior of the closed-loop DES). This system characteristic shows that the application of test functions for identification purposes like it is often done in continuous systems is not possible for industrial closed-loop DES. Only passive identification approaches working on observed system evolutions are suitable for the considered system class.

The aim of identification is to deliver a model to reproduce the external behavior of the closed-loop system. Hence, it is necessary to capture the signals exchanged between controller and plant in order to get samples of the external system behavior. In case of existing industrial facilities this process must be noninvasive to avoid disturbances of the process. In the next section, a method to capture these signals in an efficient way is presented.

\section{DATA COLLECTION}

\subsection{Technical implementation}

The implementation of a data collection procedure for closed-loop DES necessitates capturing the signals exchanged between plant and controller. The most accurate approach to get the according signal values is to connect the wires between sensors or actuators and the controller with a special data collection hardware like described in (de Smet et al., 2001). Although such an approach is possible within a laboratory environment, for existing industrial facilities the necessary cabling effort would be too important. Since one of the main reasons to use identification methods is to save costs, the effort to apply the method must not exceed the costs of manually model building. A slightly less accurate data collection approach that can be implemented with less effort is to collect the signals after they have been captured by the controller.

Figure 2 shows the functional principle of a programmable logic controller (PLC) which is a widely used class of controllers in industry. The controller cyclically performs the steps 'input reading' where it reads the signals from the sensors, 'program execution' to determine new output values for the actuators, and 'output writing' where the newly determined commands are sent to the plant actuators. Modern 
PLCs are equipped with a communication processor which makes it possible to send the values of the input and output signals to a standard PC where they can be stored in a data base. The implementation of such a connection is relatively easy (it is mainly a software problem) and does not require any special hardware.

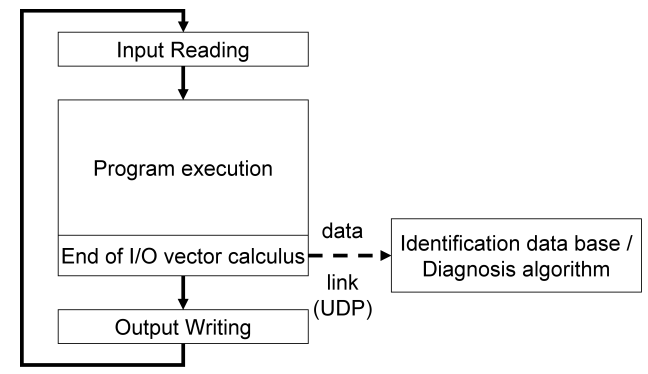

Figure 2: PLC cycle and data collection

As implementation of the data link between controller and identification data base, a UDP (User Datagram Protocol) connection is used. At the end of the 'program execution' phase, the communication processor of the controller sends a UDP-datagram which is received by the $\mathrm{PC}$ with the identification data base. In order to validate that this connection is fast enough to be used for data collection, tests have been performed using a Siemens PLC (CPU 315-2 DP) equipped with a program leading to a PLC-cycle time of 25 to $30 \mathrm{~ms}$. The PLC as a communication processor (CP 343-1 IT) which sends the data to a standard PC (identification data base). Figure 3 shows that the time between the reception of two packages alters between $25 \mathrm{~ms}$ and $30 \mathrm{~ms}$ according to the slightly varying PLC-cycle. It could also be observed that no data packets got lost during the transmission. This shows that the UDP connection is adapted for our purposes.

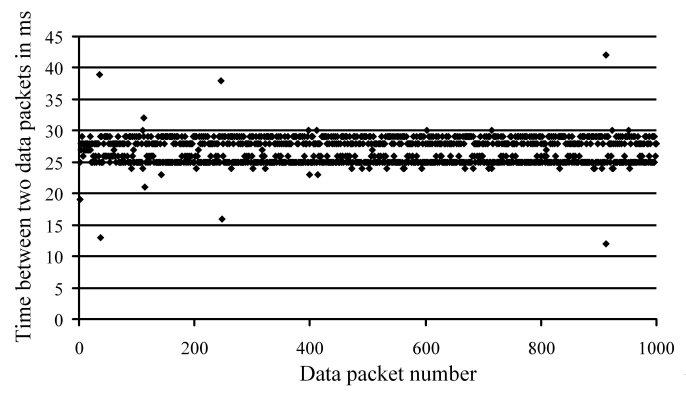

Figure 3: Validation of the UDP connection

When the signal values are sent to the identification data base, they are grouped in the controller I/O (input/output) vector defined as follows:

Definition 1 (Controller I/O vector). Given $r$ different controller inputs $I_{1}, \ldots, I_{r}$ and $s$ different controller outputs $O_{1}, \ldots, O_{s}$, the controller $I / O$ vector $u=\left(I O_{1}\right.$, $\left.\ldots, I O_{m}\right)$ with $m=r+s$ is given by $I O_{i}=I_{i} \forall i=$ $1, . ., r$ and $I O_{r+1}=O_{i} \forall i=1, . ., s . m=|u|$ denotes the length of the vector (number of controller I/Os).

The controller I/O vectors which are sent to the identification data base differ slightly from the real values of the signals. Three scenarios are considered to describe how the controller $\mathrm{I} / \mathrm{O}$ vector captured in the data base is affected by the data collection process at the end of the 'program execution' step in the PLC.

Figure 4 shows the evolution of two controller inputs (sensor values) in the plant. It can be seen that the two signals change their values at different times since the according sensors are not triggered simultaneously. In the middle of the figure, the PLC-cycle is shown over time. The dotted line indicates that the input values of the plant are read by the PLC during the step 'input reading'. After the program execution phase, the input values are sent to the identification data base. The evolution of the data received in the data base (the sampled data) is shown below the PLC cycle. Since the real values of the two inputs have been captured simultaneously during the 'input reading' phase they also change their value simultaneously in the identification data base. As a consequence for the identification algorithm it is important to use an appropriate definition of the notion event. Since in DES theory events cannot occur simultaneously it is not possible to define the change in value of one signal as an event like it would probably be the most intuitive way. Instead we use the following definition:

Definition 2 (Event). The appearance of an event leads to a new I/O vector $u(j)$ with $u(j) \neq u(j-1)$. Only I/O vectors generated by events are stored in the data base.

As a consequence of the this definition, two successive $\mathrm{I} / \mathrm{O}$ vectors $u(j)$ and $u(j+1)$ always differ in at least one (but possibly more than one) I/O value.

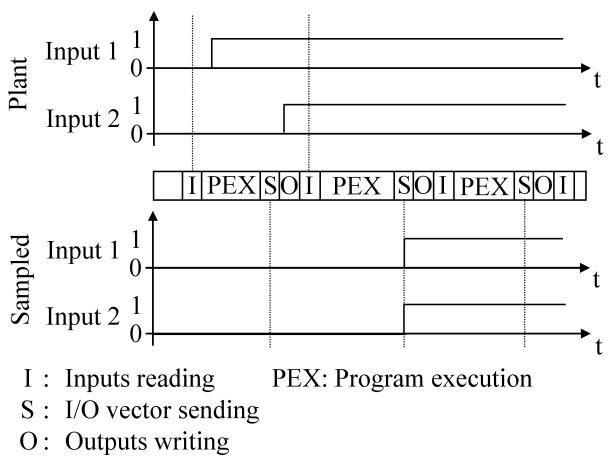

Figure 4: Sampling scenario 1 
In figure 5 a scenario with a controller input and a controller output is shown. In the example it is assumed that there is a logical condition in the control algorithm relating these two signals: if the input changes its value, the controller changes the value of the output as a consequence. It can be seen that the cause (change in value of the input) and the according effect (change in value of the output) are sent simultaneously to the data base. Hence, in the data base cause and effect cannot not directly be seen. Additionally, the figure shows that using the described data collection procedure it is possible to receive $\mathrm{I} / \mathrm{O}$ vectors in the data base before the according output values are valid for the plant. The output values are sent to the plant during the step 'output writing' which takes place after the transfer of the $\mathrm{I} / \mathrm{O}$ vector.

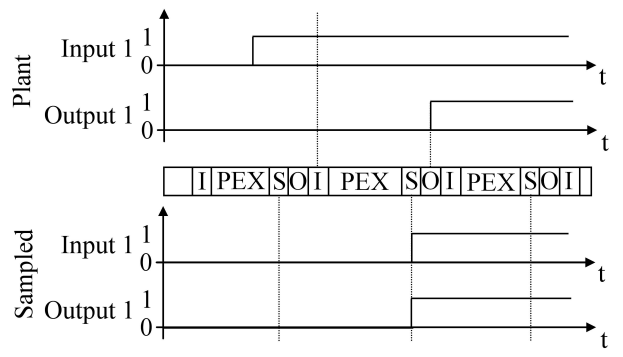

Figure 5: Sampling scenario 2

The third scenario in figure 6 shows the case of an actuator influencing a sensor in the plant. When the according output is set in the controller it is transfered to the data base and with a short delay written to the the plant. The actuator controlled by the output can only then start influencing the sensor connected with input 1 . Hence, in this case cause and effect cannot be captured simultaneously since the change in value of input 1 occurs some time after the vector with the change in value of output 1 has been sent to the identification data base. The delay of cause and effect is often not considered in manually built models like in (Sampath et al., 1996). A second issue of the data collection process can also be seen in the figure: although input 1 and output 1 change their value relatively fast one after the other (faster than the duration of a PLC-cycle), it can take up to one PLC cycle until this change in value is sent to the identification data base.

The three scenarios show that the selected data collection procedure introduces some inadequacies that will also be part of the identified model. It is thus important to clearly indicate when the data has been captured in the PLC-cycle to precisely describe the data in the identification data base.

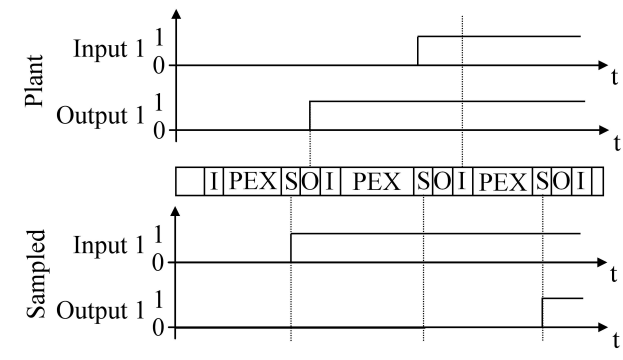

Figure 6: Sampling scenario 3

\subsection{Definition of the observed language}

For identification, the captured system data can be interpreted as the language of the considered closedloop DES. The identification is based on the observation of I/O vector sequences during $l_{h}$ different system evolutions:

Definition 3 (I/O vector sequence). If during the $h$-th system evolution $l_{h} I / O$ vectors $u_{h}$ have been observed, the sequence is denoted as $\sigma(h)=\left(u_{h}(1)\right.$, $\left.u_{h}(2), \ldots, u_{h}\left(l_{h}\right)\right)$.

The term 'system evolution' refers to a system run of a certain length. In manufacturing systems such an evolution can be a production cycle. Based on the $\mathrm{I} / \mathrm{O}$ vector sequences it is possible to define the observed word set (I/O vector sequences of a given length) and the observed language:

Definition 4 (Observed word set and language). The observed words of length $q$ captured during $p$ different system evolutions are denoted as

$W_{\text {Obs }}^{q}=\bigcup_{i=1}^{p}\left(\bigcup_{j=1}^{l_{i}-q+1}\left(u_{i}(j), u_{i}(j+1), \ldots, u_{i}(j+q-1)\right)\right)$.

With the observed word set we can define the observed language of length $n$ of the system starting from any reachable state as

$$
L_{O b s}^{n}=\bigcup_{i=1}^{n} W_{O b s}^{i}
$$

In most practical applications the observed system language is only a subset of the possible system language $L_{\text {Orig }}^{n}$. The longer a closed loop system is observed, the more likely the cardinality of $L_{O b s}^{n}$ converges to a certain value. If new system evolutions do not lead to new words in $L_{O b s}^{n}$, the system language $L_{\text {Orig }}^{n}$ can reasonably be considered as completely observed $\left(L_{O b s}^{n} \approx L_{\text {Orig }}^{n}\right)$. Figure 7 shows typical evolutions of the observed language in case of convergence and in case of continued growth. In practical application it is often the case that $L_{O b s}^{n}$ converges for smaller 
values of $n$ but still grows for larger values. As an example consider the case when each possible single system output has been observed ( $L_{\text {Obs }}^{1}$ converges) but there still occur new combinations (sequences) of already known single system outputs ( $L_{O b s}^{n>1}$ continues to grow).

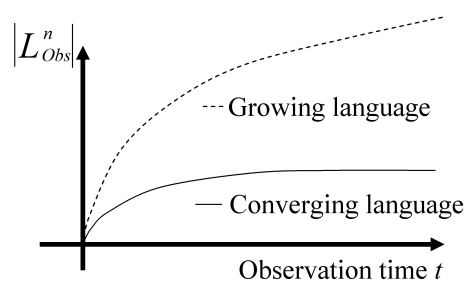

Figure 7: Principle of a converging language

\section{IDENTIFICATION}

The aim of identification is to build a model that approximates the original system language $L_{\text {Orig }}^{n}$. In (Klein, 2005) a non-deterministic autonomous automaton with output is chosen as an appropriate model to reproduce the observed language of closedloop discrete event systems:

Definition 5 (Non Deterministic Autonomous $\mathrm{Au}$ tomaton with Output). NDAAO $=\left(X, \Omega, r, \lambda, x_{0}\right)$ with $X$ finite set of states, $\Omega$ output alphabet, $r: X \rightarrow$ $2^{X}$ non-deterministic transition relation, $\lambda: X \rightarrow \Omega$ output function and $x_{0}$ the initial state.

If the output alphabet $\Omega$ consists of the captured $\mathrm{I} / \mathrm{O}$ vectors it is possible to approximate the observed language $L_{O b s}^{n}$ by performing state trajectories in the automaton:

Definition 6 (Words and Language of the NDAAO). The set of words of length $n$ generated from a state $x(i)$ is defined as:

$$
W_{x(i)}^{n=1}=\left\{w \in \Omega^{1}: w=\lambda(x(i))\right\}
$$

and

$$
\begin{array}{r}
W_{x(i)}^{n>1}=\left\{w \in \Omega^{n}: w=(\lambda(x(i)), \lambda(x(i+1)),\right. \\
\ldots, \lambda(x(i+n-1))): x(j+1) \in r(x(j)) \\
\forall i \leq j \leq i+n-2\}
\end{array}
$$

The language of length $n$ generated by the NDAAO is given by

$$
L_{\text {Ident }}^{n}=\bigcup_{i=1}^{n} \bigcup_{x \in X} W_{x}^{i}
$$

In (Klein, 2005) an algorithm to identify an NDAAO based on an observed language is given. The algorithm delivers a model that is $k+1$-complete which means that $L_{\text {Ident }}^{k+1}=L_{\text {Obs }}^{k+1}$ (proof can be found in (Klein, 2005)). This property excludes that the identified model contains any non-observed word of length $k+1$. This makes the model suitable for fault detection purposes (Roth et al., 2009). It is also the basis for the completeness of the model which will be shown at the end of the section. The algorithm uses words of the parametric length $k$ to construct the NDAAO. The observed I/O vector sequences in the data base have to be modified according to the following equation. It duplicates the first vector of each sequence $k-1$ times:

$$
\sigma_{h}^{k}(i)=\left\{\begin{array}{l}
\sigma_{h}(1) \text { for } 1 \leq i \leq k \\
\sigma_{h}(i-k+1) \text { for } k<i \leq k+\left|\sigma_{h}\right|-1
\end{array}\right.
$$

On the basis of $\Sigma^{k}=\left\{\sigma_{1}^{k}, \ldots, \sigma_{p}^{k}\right\}$ we determine the observed word sets of length $k$ and $k+1$ for the identification:

$$
W_{O b s, \Sigma^{k}}^{k}=\bigcup_{\sigma_{h}^{k} \in \Sigma^{k}}\left(\bigcup_{i=1}^{\left|\sigma_{h}^{k}\right|-k+1}\left(u_{h}(i), u_{h}(i+1), \ldots, u_{h}(i+k-1)\right)\right)
$$

$$
W_{O b s, \Sigma^{k}}^{k+1}=\bigcup_{\sigma_{h}^{k} \in \Sigma^{k}}\left(\bigcup_{i=1}^{\left|\sigma_{h}^{k}\right|-k}\left(u_{h}(i), u_{h}(i+1), \ldots, u_{h}(i+k)\right)\right)
$$

The identification procedure is given in algorithm 1. It is a condensed version of the algorithm given in (Klein, 2005). For the algorithm, we define an operator $w[a . . b]$ to deliver the substring from position $a$ to position $b$ in word $w$. In the first step, for each word of length $k$ a state is created. If two words of length $k$ have been observed successively, they build a word of length $k+1$. Hence, the states representing the two words of length $k$ are connected in step 2 of the algorithm. In step 3, the output function of each state is redefined. The new state output is the $\mathrm{I} / \mathrm{O}$ vector at the end of the word of length $k$ representing the state output so far. In the last step, states with equal output and equal following states are merged. A detailed description of this procedure is given in (Klein, 2005).

An interesting characteristic of a model identified with algorithm 1 is that under certain conditions the identified language does not only reproduce the system behavior observed so far $\left(L_{\text {Ident }}^{n} \supseteq L_{\text {Obs }}^{n}\right)$ but the complete original system language $\left(L_{\text {Ident }}^{n} \supseteq L_{\text {Orig }}^{n}\right)$. This property (which is not proved in (Klein, 2005)) is very important for many model-based techniques relying on a complete system description. In the fol- 


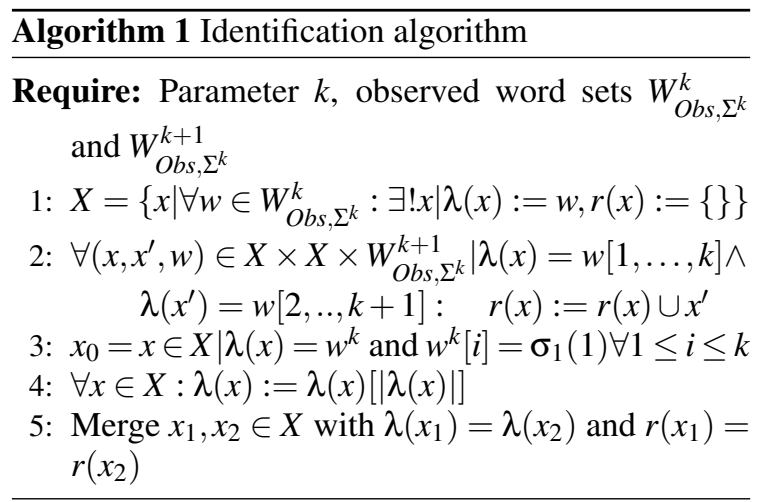

lowing, the necessary conditions to proof the characteristic are given. The first step is to show that each state trajectory producing a word of length $k$ ends in the same state. In the following, $w^{k}$ denotes a word of length $k$.

Lemma 1. In an NDAAO identified with parameter $k$ each state trajectory $\lambda(x(i), \ldots, x(i+k-1))=w^{k} \in$ $L_{\text {Ident }}^{k} \mid x(j+1) \in r(x(j)) \forall i \leq j<i+k-1$ ends in the same state.

We define a function $\widetilde{\lambda}(x)$ delivering $w^{k}$ used in step 1 for each state of the algorithm. It represents the state output before it has been replaced by the I/O vector at the end of the word of length $k$ representing the state output until step 3 .

Proof of lemma 1. From equations 1 and 2 it follows that $\forall w^{k} \in L_{O b s}^{k} \exists v_{1}^{k}, \ldots, v_{k}^{k} \in W_{O b s, \Sigma^{k}}^{k} \mid v_{1}^{k}[k]=$ $w^{k}[1], v_{2}^{k}[k-1 \ldots k]=w^{k}[1 \ldots 2], \ldots, v_{k}^{k}[1 \ldots k]=$ $w^{k}[1 \ldots k]$. From steps 1 and 2 of algorithm 1 it follows that states representing $v_{1}^{k}, \ldots, v_{k}^{k}$ are connected since $\forall v_{1}^{k}, v_{2}^{k} \exists w^{k+1} \in W_{O b s, \Sigma^{k}}^{k+1} \mid w^{k+1}=v_{1}^{k}[1 \ldots k] v_{2}^{k}[k]$.

Since step 1 assures that $\forall w^{k} \in L_{\text {Ident }}^{k} \exists ! x \mid \tilde{\lambda}(x)=w^{k}$ it follows that each state trajectory with $\lambda(x(i), \ldots, x(i+k-1))=w^{k} \in L_{\text {Ident }}^{k} \mid x(j+1) \in$ $r(x(j)) \forall i \leq j<i+k-1$ ends in the same state.

In the next theorem, it is stated that the identified language simulates the original system language of arbitrary length if $L_{O r i g}^{k+1}=L_{O b s}^{k+1}$ holds for a given value of the identification parameter $k$.

Theorem 1. If $L_{\text {Orig }}^{k+1}=L_{\text {Obs }}^{k+1}$, then $L_{\text {Ident }}^{k+n} \supseteq L_{\text {Orig }}^{k+n}$ for an NDAAO identified with parameter $k$.

Proof of theorem 1. $L_{\text {Ident }}^{k+1} \supseteq L_{\text {Orig }}^{k+1}$ since the identified NDAAO is $k+1$ complete. For $k+2$ it holds: $\forall w^{k+2} \in L_{\text {Orig }}^{k+2} \exists a^{k} b^{1} c^{1}=d^{1} e^{1} f^{k}=s^{1} u^{k} v^{1}=$ $w^{k+2} \mid a^{k} b^{1}, e^{1} f^{k}, u^{k} v^{1} \in L_{\text {Orig }}^{k+1}=L_{O b s}^{k+1}$. Each state trajectory producing $a^{k}$ ends in the same state $x_{1}$ (lemma $1)$. In step 2 of the algorithm, this state is connected with $x_{2} \mid \widetilde{\lambda}\left(x_{2}\right)=u^{k}$. Each state trajectory producing $u^{k}$ ends in the same state $x_{2}$ which gets connected to $x_{3} \mid \widetilde{\lambda}\left(x_{3}\right)=f^{k}$. Since there is a trajectory leading to state $x_{1}$ and $x_{1}, x_{2}$ and $x_{3}$ are in one trajectory, it follows that $\forall w^{k+2} \in L_{\text {Orig }}^{k+2}$ there exists a trajectory of states producing this word. For larger values than $k+2 \forall w^{k+n} \in L_{\text {Orig }}^{k+n}$ there is always an appropriate decomposition into already observed substrings of $L_{\text {Orig }}^{k+1}=L_{O b s}^{k+1}$ to find a trajectory of connected states like presented above. Hence, it follows that $L_{\text {Ident }}^{k+n} \supseteq L_{\text {Orig }}^{k+n}$ if $L_{\text {Orig }}^{k+1}=L_{\text {Obs }}^{k+1}$ holds.

Theorem 1 shows that it is crucial to state $L_{\text {Orig }}^{k+1}=$ $L_{O b s}^{k+1}$ for a precise $k$ in order to deliver a model which is able to simulate the complete original system behavior. In section 3.2 it is shown how it can be decided if $L_{\text {Orig }}^{k+1}=L_{O b s}^{k+1}$ holds for a given value of $k$.

\section{APPLICATION}

In order to show that the identification algorithm of section 4 is capable of delivering a model of existing systems that can be interpreted as closed-loop DES, one of the case studies we have treated will be presented in this section. The system depicted in figure 8 has 30 digital I/Os and is controlled by a Siemens PLC equipped with a communication processor. The system treats work pieces stored in the left most part of the facility. Each work piece is successively treated by the three tools. For identification a system evolution is defined as the run of two work pieces through the machine. Hence, there are at most two work pieces treated concurrently in the whole system.

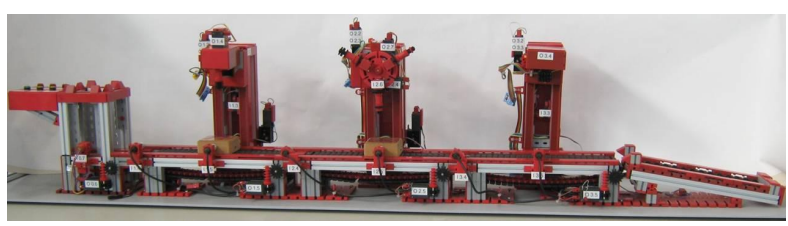

Figure 8: Laboratory facility

50 system evolutions (treating 50 times two work pieces) have been performed and the according data has been collected using the procedure described in section 3. The observed word sets of different length are shown in figure 9. It can be seen that for small values of $n$ like $n=2$ or $n=3$ the according observed word set and thus the observed language converges to a stable level which implies that the observed language converges to the original system language $\left(L_{O b s}^{n=3} \approx L_{\text {Orig }}^{n=3}\right)$. Although for $L_{O b s}^{3}$ there is a new word observed in one of the last evolutions, it 
can still reasonably be considered as completely observed. Hence, the precondition of theorem 1 is fulfilled. Since $L_{\text {Obs }}^{n=3} \approx L_{\text {Orig }}^{n=3}$, it is possible to identify an NDAAO with $k+1=n=3(\rightarrow k=2)$ to simulate the original system behavior.

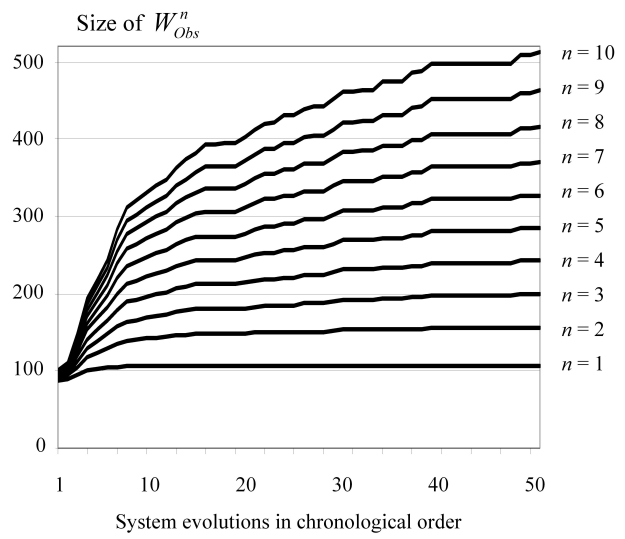

Figure 9: Observed word set for the laboratory system

For the identification of an NDAAO a software tool has been developed. Like depicted in figure 10, it takes the data base consisting of the observed system evolutions and the identification parameter $k$ as input and applies algorithm 1. The software allows an analysis of the model structure (number of states, number of transitions etc.) and a behavioral analysis (see below). The identified model can be exported to an XML-file to use it in other tools such as diagnosis software (Roth et al., 2009). To visualize the resulting automaton, an interface to GRAPHVIZ is provided. The identification of the NDAAO with $k=2$ on the basis of 50 system evolutions took $170 \mathrm{~ms}$ on a standard PC with a $1.79 \mathrm{Ghz}$ CPU and 1.96 GB RAM. The identified model has 121 states and 162 transitions.

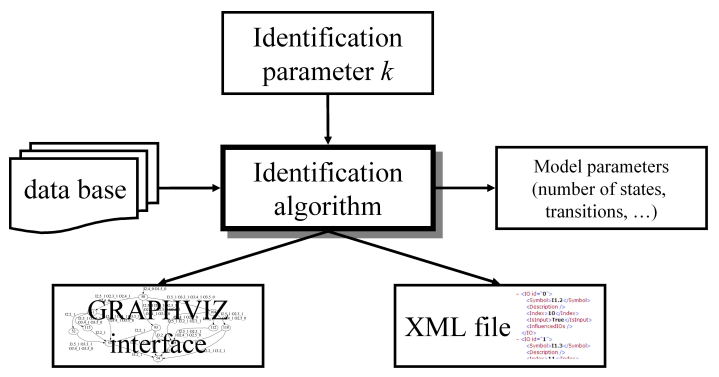

Figure 10: Features of the implemented software

A part of the identified NDAAO can be seen in figure 11. Due to space limitations, instead of giving the complete $\mathrm{I} / \mathrm{O}$ vector in each state output, only I/Os changing their value from one state to another are given (e.g. I2.4_1 O2.5_1 to indicate a rising edge
(_1) of controller Input $I 2.4$ and a rising edge of controller Output $O 2.5$ from state 48 to state 49 ). Several examples for the different I/O vector sampling scenarios presented in section 3.1 can be seen in the model. For the scenario with two controller inputs changing their value synchronously due to the data collection process (figure 4), the transition between states 93 and 54 is an example. Both inputs $I 2.2$ and $I 3.2$ change their value when taking this transition. The transition from state 48 to 49 is an example for the second sampling scenario (figure 5) where the change in value of an input triggers the change in value of an output. This transition represents the situation when the second work arrives at the entrance of the second station (position sensor connected to $I 2.4$ changes its value) and the conveyor of this station is started (controller output $O 2.5$ is set to 1). Both changes in value appear at one transition due to the data collection process as explained in section 3.1. An example for the third scenario form section 3.1 (figure 6) can be seen in the state trajectory $x_{48} \rightarrow x_{49} \rightarrow x_{50}$. From state 48 to state 49 the conveyor is started (O2.5_1) to transport the work piece away from the entrance position (I2.5). Since it takes at least until the next PLC cycle to transport the work piece away from the sensor (I2.5_0 for the falling edge) cause and effect cannot be seen at once but at some successive transitions. The non-deterministic nature of the identified NDAAO can also be seen in figure 11: there are several ways to go from state 50 to state 55 . Being in state 50 the choice of the trajectory is not determined but taken at random like in the closed-loop DES where unpredictable physical conditions in the plant lead to non-deterministic behavior.

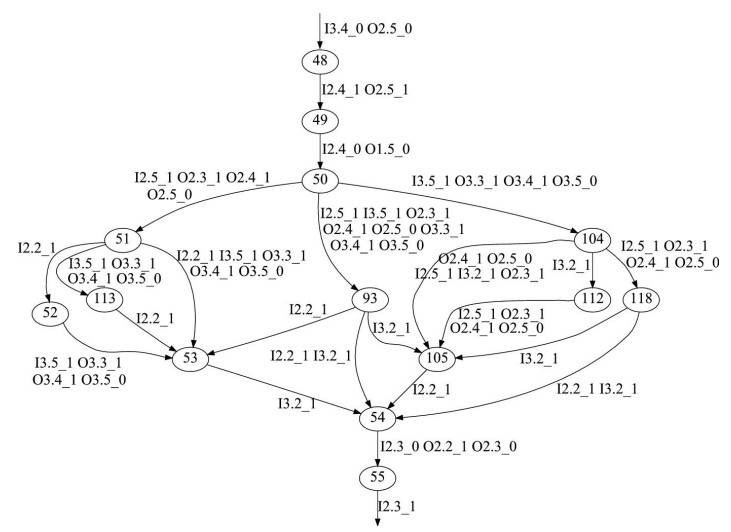

Figure 11: Part of the identified NDAAO

One of the important characteristics of the identified model is its capability to simulate the complete original system language (theorem 1). Even if only a subset of possible $\mathrm{I} / \mathrm{O}$ vector sequences connecting 
the closed-loop system states represented by NDAAO states 50 and 55 has been observed, the model contains each possible trajectory that can occur when going from one state to the other as long as no new word of length $k+1$ is produced. If $L_{\text {Orig }}^{k+1}=L_{\text {Obs }}^{k+1}$, the model is capable of exhibiting words of length $k+n$ although these words have not been seen before. This capability comes at cost of also exhibiting words that have not and probably will not be observed $\left(w^{k+n} \notin L_{\text {Orig }}^{k+n}\right.$ ). To get some information of the amount of words which are created without having been observed, the coefficient

$$
C_{B}^{n}=\frac{\left|L_{\text {Ident }}^{n}\right|}{\left|L_{\text {Obs }}^{n}\right|}
$$

is a useful indicator. The presentation of the coefficient in figure 12 describes the relation between the identified and the observed language of an automaton identified with $k=2$. For $n=k+1$, the model strictly creates the observed language $L_{O b s}^{k+1}$. It can be seen that for larger values of $n$ the automaton generates a larger language than $L_{O b s}^{n}$. A certain part of the additionally created words is probably not part of the original system language which may lead to a need for specific precautions in some model based techniques like diagnosis. However, from theorem 1 it is clear that each word with length $n \geq k+1$ of the original language not observed so far is part of the identified language. In the case of model based diagnosis for example, this allows stating that there will be no false alerts using the identified automaton as fault-free reference model (Roth et al., 2009).

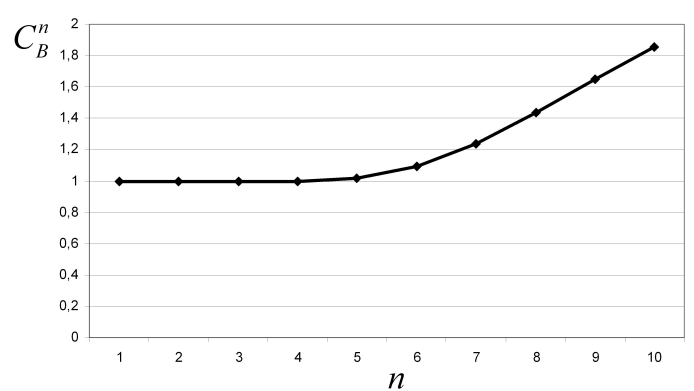

Figure 12: Coefficient of identified and observed language

\section{CONCLUSION}

In this paper practical implications of identification of closed-loop discrete event systems have been addressed. It has been shown how the necessary data can be obtained in the case of industrial closed-loop systems. For many model-based techniques it is crucial to have a model of the complete system behavior. For the identification algorithm of (Klein, 2005) it has been proved that the identified automaton is simulates the original system language of arbitrary length if some conditions concerning the observed system language hold.

\section{REFERENCES}

Biermann, A. and Feldman, J. (1972). On the synthesis of finite-state machines from the sample of their behavior. IEEE transactions on computers, 21:592-597.

de Smet, O., Denis, B., Lesage, J.-J., and Roussel, J.-M. (2001). Dispositif et procd d'analyse de performances et d'identification comportementale d'un systme industriel en tant qu'automate vnements discrets et finis. Technical report, French Patent 01110933.

Dotoli, M., Fanti, M. P., and Mangini, A. M. (2006). Online identification of discrete event systems: a case study. In 2006 IEEE international conference on automation science and engineering, pages 405-410.

Fanti, M. P. and Seatzu, C. (2008). Fault diagnosis and identification of discrete event systems using petri nets. In Proceedings of the 9th International Workshop on Discrete Event Systems, Gtebor, Sweden, pages 432435.

Giua, A. and Seatzu, C. (2005). Identification of freelabeled petri nets via integer programming. Proceedings of the 44th IEEE Conference on Decision and Control, and the European Control Conference 2005 Seville, Spain, December 12-15, 2005, pages 76397644.

Klein, S. (2005). Identification of Discrete Event Systems for Fault Detection Purposes. Shaker Verlag.

Klein, S., Litz, L., and Lesage, J.-J. (2005). Fault detection of discrete event systems using an identification approach. In Proceedings of the 16th IFAC World Congress, pages CDROM paper n02643, 6 pages.

Machado, J., Denis, B., and Lesage, J. J. (2006). A generic approach to build plant models for DES verification purposes. In Proceedings of the 8th international workshop on discrete event systems, pages 407-412.

Meda-Campana, M. and Lopez-Mellado (2005). Identification of concurrent discrete event systems using petri nets. 2005 IMACS: Mathematical Computer, Modelling and Simulation Conference.

Roth, M., Lesage, J.-J., and Litz, L. (2009). An FDI method for manufacturing systems based on an identified model. In Proceedings of the 13th IFAC Symposium on Information Control Problems in Manufacturing, INCOM'09, pages 1389 - 1394, Moscow, Russia. IFAC.

Sampath, M., Sengupta, R., Lafortune, S., Sinnamohideen, K., and Teneketzis, D. (1996). Failure diagnosis using discrete-event models. IEEE transactions on control systems technology, 4(2):105-124. 